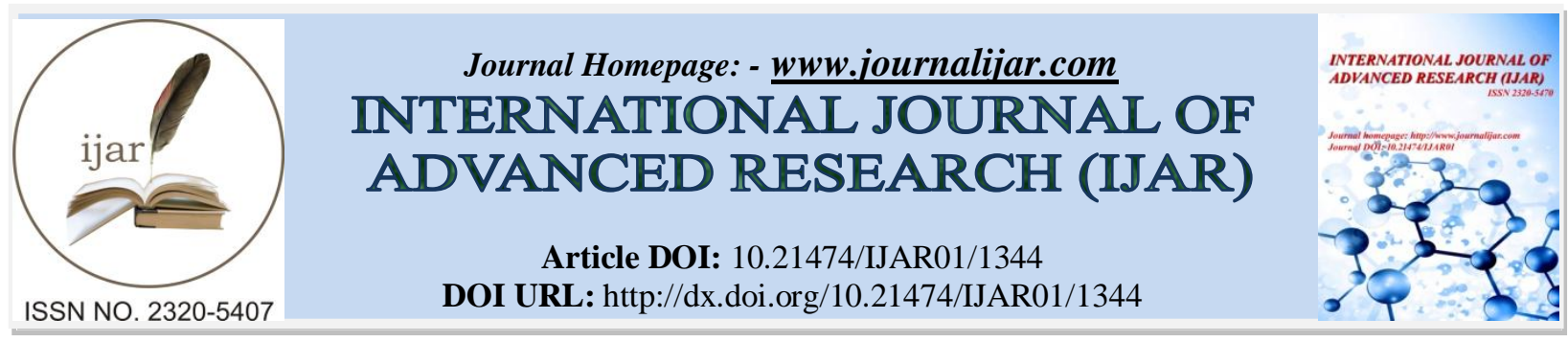

RESEARCH ARTICLE

\title{
ONLINE SHOPPING: MOTIVATORS AND TRIGGERS TO COMPULSIVE E-TAILING.
}

\author{
Rishabh Kapur ${ }^{1}$ and Dr. Surbhi Kapur ${ }^{2}$. \\ 1. MBA Scholar, NMIMS, Mumbai, India. \\ 2. Associate Professor, KIIT School of Management, KIIT University, Bhubaneswar, India.
}

\section{Manuscript Info}

Manuscript History

Received: 12 June 2016

Final Accepted: 19 July 2016

Published: August 2016

Key words:-

Online shopping, motivating factor, ads, discounts, compulsive buying

\begin{abstract}
Online shopping does not seem to be in its nascent phase any longer especially with the technologically savvy millennial populace taking to it head on. The present paper tries to delve deeper into online shopping behavior, the chief determinant of online buying, perceptions regarding multiple aspects of online shopping and the association with gender, age, occupation thereof. Moreover, it sheds light on how online shopping becomes a potent platform for compulsive buying and how the social media ads and discounts force him to buy products which may not be in his or her shopping list. A survey was conducted on 153 online shopping site users revealing online buying as the new choice and pricing not convenience as the major motivating factor. Cross tabulation procedure along with Chisquare test of association was used to study the frequency, relative frequency and test of association.
\end{abstract}

Copy Right, IJAR, 2016,. All rights reserved.

\section{Introduction:-}

Shopping has always been an interesting and integral part of our lives. Some call it a necessity, others a stress buster and still others a waste of time. Yet, this fascinating experience retains its importance down the ages. From the barter system to the modern day retailing and now e-tailing, it changes its character and yet basically remains the same activity - buying. The most dramatic change that recent times has ushered are the online shopping sites which have simply changed the way people used to shop. Visiting far away outlets and malls to shop can be an annoying effort due to the increasing traffic in cities and the weather which might not always support the decision of going out. This coupled with the increasing popularity and convenience of e-commerce sites has changed the shopping phenomenon to a great degree. Now you can shop with just a click of a key on your iPad or laptop in the tranquil surroundings of your home and can choose from hundreds of brands and thousands of products at reasonable prices and attractive discounts. With internet being a major part of our lives, we have become dependent on it for a multitude of services. Online shopping too has witnessed a sudden surge in its users and popularity. According to a study jointly conducted by The Associated Chambers of Commerce and Industry of India (ASSOCHAM) and Grant Thornton, the "changing lifestyle of the urban population has also resulted in many people relying on the internet for their shopping needs". Shopping from the comfortable confines of your home with larger product variety at your disposal has ushered an increased dependence on the online platform (Thornton, 2015). Much ink has been spilt on how e-commerce sites have eased the shopping experience and about its benefits and detriments. The endeavour here is to add to the existing research by revisiting online shopping behaviour pattern, determining the prime factor of online purchase and discerning the perception of people on various aspects of online shopping and their association with age, gender and occupation. Additionally it tries to explore whether social media ads and discounts 
coerce the consumer to purchase products even if they don't need it making it a possible breeding ground for compulsive buying. The following sections include a relevant review of literature, methodology, results, discussion and conclusion.

\section{Review of Literature:-}

Buying online has its advantages like the range it offers. Inadequate shelf space is not a constraint what with a wider array of products available online. Another benefit is convenience as delivery of the product at your doorstep takes care of the limiting factors like timing, driving and parking, more so for working people who are constantly rushing against time. Finally, matchless prices owing to lower operational costs as compared to offline stores is also a bonus. The popularity and possibilities of online shopping with all its ease, availability as well as utility has triggered ample research on online buying behaviour. Some suggest that brand pursuers are inclined to buy online as they might not see a risk involved in buying established brands" (Lunn and Suman 2002). Others opine that price can be an instrument of motivation when consumers find reduced prices or pecuniary advantages exhibited on online shopping portals (Sim and Koi 2002). Similarly, a survey conducted on 325 Indian students reveals that buying online is "influenced by utilitarian value, attitude toward online shopping, availability of information, and hedonic values" (Khare and Rakesh 2011). The findings also indicate that male students show a "more positive attitude toward online shopping" in comparison to their female counterparts which has also been indicated by prior research (Khare and Rakesh 2011; Haque et al. 2007; Cho and Jialin 2008; Hashim et al. 2009; Hasan 2010; Chou et al. 2010). Surprisingly some research hints that price may not be a key motivator. Out of the four factors "namely Anxiety, Ease of Use, Usefulness and Price as the determinants of shopper buying behaviour online", anxiety figures as the "most important factor in online shopping though the shoppers are young, technology savvy and prefer buying online". Then comes 'Ease of use' "followed by 'Usefulness' and 'Price'". Therefore online shoppers may be buying " more for convenience and utility than for price difference or discounts." (Panda and Swar, 2014). Studies also show that there is a direct relationship between the expertise a person has of online shopping and his buying behaviour (Lee and Park). Whatever the reason, the Grant Norton report predicts that the "Indian e-commerce market would grow at a Compounded Annual Growth Rate (CAGR) of 63\% to reach USD 8.5 billion in CY $2016 "$. It adds that online shoppers would "increase from 20 million in 2013 to 40 million in 2016".

\section{Compulsive Buying:-}

The increase in online shoppers is definitely visible and online shopping for food, apparel, grocery, shoes, mobiles and accessories has grown considerably and continues to amplify. To the extent that it sometimes borders on becoming an addiction quite like compulsive buying which implies "a consumer's tendency to be preoccupied with buying that is revealed through repetitive buying and a lack of impulse control" (Ridgway, Kukar-Kinney \& Monroe, 2008). Compulsive buying can also be defined as " excessive buying or out-of-control buying" ( Lee \& Park; Faber, O'Guinn, and Krych 1987). With its distinctive features like easy access, huge product line and striking online exhibits, online shopping could well augment online compulsive buying ( Lee \& Park; Eastin 2002). As "many compulsive buyers want to avoid being reminded of normative standards by important others", online shopping can be an excellent way for them "to shop in isolated shopping environments" (Lee \& Park). Further, it has been supposed that variations in consumer culture could induce an increase in compulsive buying among consumers (Neuner et al., 2005). With the changing consumer culture, shopping has moved beyond fulfilling physical wants to being a source of joy and recreation (Millan \& Howard, 2007), a means of communicating one's individuality (Dittmar, Long \& Meek, 2004) and achieving social standing (Han, Nunes \& Drèze, 2010). The same can hold true for online compulsive buying by online shoppers what with "75\% of Indian internet users" being in "the age group of 15 to 34 years" who shop "more than any other category" owing to "peer pressure, rising aspirations with career growth, and fashion trends" (Grant Thorton, 2015)

\section{Social Ads and Discounts:-}

Internet has eased the way for customers to shop, but the darker side to it is the way it compels the customers to buy something by increasing the number of ads on social media or by the discounts offered. The shopping websites prod us constantly toward their shopping carts and a dress/pair of shoes which you must have seen once keeps popping up every time you check your facebook "like a stealth shopping mousetrap". Does this "cue-reactivity" or stimulation from shopping signals or cues lead to a "craving" to buy? (Marlynn Wei M.D., J.D. 2015). Similarly, sometimes discounts or minimum cart amount to be fulfilled to avail a discount triggers purchase of additional products which might not be really necessary or may be absent in the original list. Infact, Mintel's Online Shopping US 2015 report reveals that $48 \%$ of American online shoppers confess to intermittently "increasing the size of their orders to 
hit the free shipping minimum". Despite claiming to select deals with lowest prices, "consumers are averaging $\$ 114$ per online order, driving online retail sales from $\$ 264.2$ billion in 2013 to $\$ 304.9$ billion in 2014". Therefore, free shipping proves to be an impetus for online shoppers to meet or exceed the "free shipping threshold". The report further states that "over two thirds (69 percent) of US online adults shop online at least monthly, with 33 percent shopping online every week in 2015, up from 24 percent in 2014". An Accenture survey (2013) revealed that "as online shopping continues to grow as a consumer preference" a symbiotic association between offline stores and online platforms develops. It was found that $73 \%$ of respondents indulged in "showrooming', or browsing at least once in-store and then buying online". As opposed to this, $88 \%$ disclosed that they " participated in "webrooming", or browsed online first and then bought offline. Moreover, the survey findings indicated that "43 percent of all U.S. consumers plan to shop more online and 23 percent plan to shop more with their mobile phones in the future".

This symbiotic connection spills over to the offline effect of online ads and a Harvard study states that " online ads have a powerful effect on off-line sales" (Abraham. M, 2008)

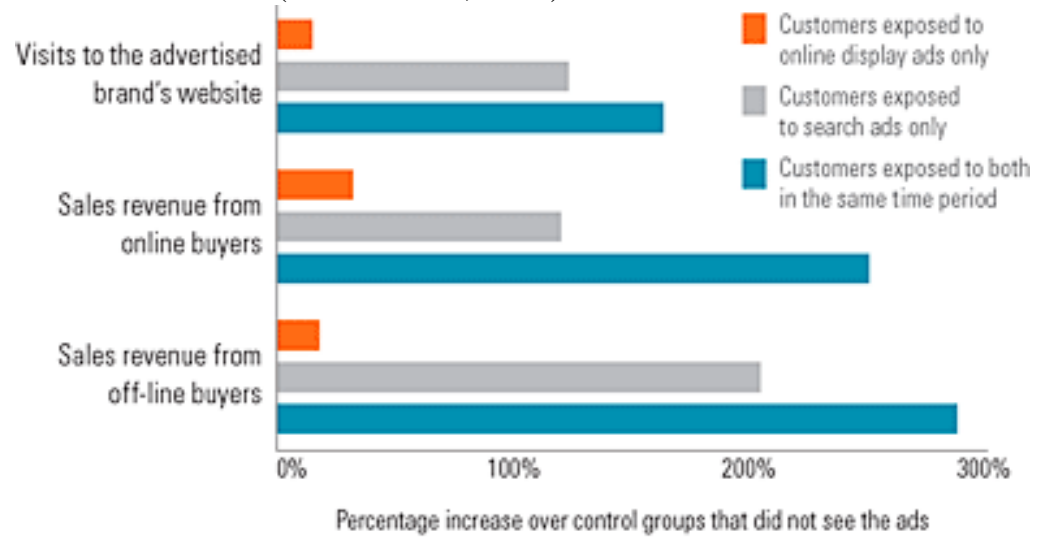

Source comscore

It further adds that independently, "search ads and display ads have the power to drive purchases, but they get the best results when used together in a single campaign" (Abraham. M, 2008). If online ads can have a considerable effect on offline sales, it could have some effect on online sales as well. Having said that and with limited research on how the social media ads and discounts motivate a consumer to buy products which may not be in his or her shopping list and whether this could lead to compulsive buying, this study tries to probe further into this.

\section{Methodology:-}

Keeping in view the objective of the research both secondary and primary data were used. A comprehensive review of literature on existing research set the ground for further inroads into a more structured analysis of the issue. The primary research instrument was designed after consultation with active online shoppers who had first-hand experience of regular online shopping and from relevant secondary data. A total of 25 questions including the demographics were included in the questionnaire but after an initial pilot study where the questionnaire was sent to 30 respondents only 19 were retained. The Cronbach Alpha reliability for the instrument was tested and the value was found to be 7 , which is acceptable. The sample was selected based on random sampling procedure from the database of current and old alumni of MBA students to capture the issues related to the objectives of this study. The questionnaire was circulated through Google forms to 200 respondents in the age group of 18 to 60 across genders out of whom 153 responded and revealed some very interesting results giving ample scope for future research in this area.

The demographic profile of the 153 respondents was evenly distributed with $48.4 \%$ males and $51.6 \%$ females. The age group 18-25 years and 26-30 years contributed $37.6 \%$ and $38.3 \%$ respondents respectively, which is much higher than the above 30 years age group (24.1\%). The major chunk of respondents $(60.8 \%)$ were working professionals and $39.2 \%$ were students. 
Table 1:- Demographic profile of study subjects.

\begin{tabular}{|c|c|c|}
\hline Age Group & No. & \% \\
\hline $18-25$ & 53 & 37.6 \\
\hline $26-30$ & 54 & 24.1 \\
\hline$>30$ & 34 & 100 \\
\hline Total & 141 & 48.4 \\
\hline Gender & \multicolumn{2}{|c|}{} \\
\hline Male & 74 & 51.6 \\
\hline Female & 79 & 100 \\
\hline Total & 153 & 39.2 \\
\hline Occupation & 60 & 60.8 \\
\hline Student & 93 & 100 \\
\hline Working Professional & 153 & \\
\hline Total & \multicolumn{2}{|l}{} \\
\hline *Total varies because of missing response
\end{tabular}

The aim of the study is to explore the online shopping behaviour pattern, ascertain the prime factor influencing the online purchase and uncover the perception of people on various aspects of online shopping and their association with age, gender and occupation. Additionally it tries to determine whether social media ads and discounts force the consumer to buy products which may be absent in their shopping list making E-tailing a conducive platform for compulsive buying. In order to achieve the above objectives, data collected as per the questionnaire was analyzed using SPSS 16 software. Cross tabulation procedure along with Chi-square test of association was used to study the frequency, relative frequency and test of association.

\section{Results:-}

Online shopping behavior:-

Table 2 throws light on online shopping behaviour where the following four questions were analyzed: i) Have you ever visited online shopping website? ii) Frequency of online shopping, iii) Do you consider returning the product if there is a delay in the expected delivery time? and iv) Have you bought anything online?

Number of respondents to each of these questions varies. Almost all, that is, $98.6 \%$ respondents had visited online shopping websites. Visit to online shopping websites was more associated with the age group of 18-25 and 26-30 years than the above 30 years category $(\mathrm{p}=0.041)$. About $40 \%$ respondents showed high or very high frequency of online shopping which did not reveal any significant association with age. Majority of the respondents $(97.1 \%)$ had online shopping experiences.

Table 2:- Online Shopping Behaviour by Age

\begin{tabular}{|c|c|c|c|c|c|c|c|c|c|c|}
\hline \multirow[t]{3}{*}{ Shopping Behaviour } & & \multicolumn{8}{|c|}{ Age Group } & \multirow{3}{*}{$\chi 2, \mathbf{p}$} \\
\hline & & \multicolumn{2}{|c|}{ 18-25 } & \multicolumn{2}{|c|}{$26-30$} & \multicolumn{2}{|c|}{$>\mathbf{3 0}$} & \multicolumn{2}{|c|}{ Total } & \\
\hline & & No. & $\%$ & No. & $\%$ & No. & $\%$ & No. & $\%$ & \\
\hline \multirow{3}{*}{$\begin{array}{l}\text { Have you ever visited an } \\
\text { online shopping website? }\end{array}$} & Yes & 53 & 100 & 54 & 100 & 32 & 94.1 & 139 & 98.6 & \multirow{3}{*}{$\begin{array}{l}\chi 2=6.385 \\
\mathrm{p}=0.041\end{array}$} \\
\hline & No & 0 & 0 & 0 & 0 & 2 & 5.9 & 2 & 1.4 & \\
\hline & Total & 53 & 100 & 54 & 100 & 34 & 100 & 141 & 100 & \\
\hline \multirow{6}{*}{$\begin{array}{l}\text { Frequency of your online } \\
\text { shopping }\end{array}$} & Very Low & 5 & 9.4 & 1 & 1.9 & 3 & 8.8 & 9 & 6.4 & \multirow{6}{*}{$\begin{aligned} \chi 2 & =12.381 \\
p & =0.260\end{aligned}$} \\
\hline & Low & 11 & 20.8 & 12 & 22.2 & 4 & 11.8 & 27 & 19.1 & \\
\hline & Medium & 18 & 34 & 19 & 35.2 & 9 & 26.5 & 46 & 32.6 & \\
\hline & High & 11 & 20.8 & 14 & 25.9 & 8 & 23.5 & 33 & 23.4 & \\
\hline & Very High & 8 & 15.1 & 8 & 14.8 & 8 & 23.5 & 24 & 17 & \\
\hline & Total & 53 & 100 & 54 & 100 & 32 & 94.1 & 139 & 98.5 & \\
\hline \multirow{3}{*}{$\begin{array}{l}\text { Do you consider returning the } \\
\text { product if there is a delay in } \\
\text { the expected delivery time? }\end{array}$} & Yes & 22 & 42.3 & 24 & 44.4 & 13 & 39.4 & 59 & 42.4 & \multirow{4}{*}{$\begin{array}{c}\chi 2=0.215 \\
p=0.898\end{array}$} \\
\hline & No & 30 & 57.7 & 30 & 55.6 & 20 & 60.6 & 80 & 57.6 & \\
\hline & Total & 52 & 100 & 54 & 100 & 33 & 100 & 139 & 100 & \\
\hline \multirow{3}{*}{$\begin{array}{l}\text { Have you bought anything } \\
\text { online? }\end{array}$} & Yes & 51 & 98.1 & 54 & 100 & 31 & 91.2 & 136 & 97.1 & \\
\hline & No & 1 & 1.9 & 0 & 0 & 3 & 8.8 & 4 & 2.9 & \multirow{2}{*}{$\begin{array}{c}\chi 2=6.112 \\
\mathrm{p}=0.047\end{array}$} \\
\hline & Total & 52 & 100 & 54 & 100 & 34 & 100 & 140 & 100 & \\
\hline
\end{tabular}


Table 3 and Table 4 present online shopping behaviour by gender and occupation. Gender and occupation did not exhibit significant association with online shopping pattern.

Table 3:- Online Shopping Behavior by Gender.

\begin{tabular}{|c|c|c|c|c|c|c|c|c|}
\hline \multirow[t]{3}{*}{ Shopping Behaviour } & \multirow[t]{3}{*}{ Response } & \multicolumn{6}{|c|}{ Gender } & \multirow{3}{*}{$\chi 2, \mathbf{p}$} \\
\hline & & \multicolumn{2}{|c|}{ Male } & \multicolumn{2}{|c|}{ Female } & \multicolumn{2}{|c|}{ Total } & \\
\hline & & No. & $\%$ & No. & $\%$ & No. & $\%$ & \\
\hline \multirow{3}{*}{$\begin{array}{l}\text { Have you ever visited an online } \\
\text { shopping website? }\end{array}$} & Yes & 72 & 97.3 & 79 & 100 & 151 & 98.7 & \multirow{3}{*}{$\begin{array}{l}\chi 2=2.163 \\
p=0.141\end{array}$} \\
\hline & No & 2 & 2.7 & 0 & 0 & 2 & 1.3 & \\
\hline & Total & 74 & 100 & 79 & 100 & 153 & 100 & \\
\hline \multirow[t]{6}{*}{ Frequency of your online shopping } & Very Low & 4 & 5.4 & 6 & 7.6 & 10 & 6.5 & \multirow{6}{*}{$\begin{array}{c}\chi 2=4.559 \\
p=0.472\end{array}$} \\
\hline & Low & 11 & 14.9 & 16 & 20.3 & 27 & 17.6 & \\
\hline & Medium & 28 & 37.8 & 27 & 34.2 & 55 & 35.9 & \\
\hline & High & 14 & 18.9 & 19 & 24.1 & 33 & 21.6 & \\
\hline & Very High & 15 & 20.3 & 11 & 13.9 & 26 & 17 & \\
\hline & Total & 72 & 97.3 & 79 & 100.1 & 151 & 98.6 & \\
\hline \multirow{3}{*}{$\begin{array}{l}\text { Do you consider returning the product if } \\
\text { there is a delay in the expected delivery } \\
\text { time? }\end{array}$} & Yes & 37 & 50.7 & 31 & 39.7 & 68 & 45 & \multirow{3}{*}{$\begin{array}{c}\chi 2=1.824 \\
p=0.177\end{array}$} \\
\hline & No & 36 & 49.3 & 47 & 60.3 & 83 & 55 & \\
\hline & Total & 73 & 100 & 78 & 100 & 151 & 100 & \\
\hline \multirow[t]{3}{*}{ Have you bought anything online? } & Yes & 71 & 95.9 & 77 & 98.7 & 148 & 97.4 & \multirow{3}{*}{$\begin{array}{c}\chi^{2}=1.139 \\
\mathrm{p}=0.286\end{array}$} \\
\hline & No & 3 & 4.1 & 1 & 1.3 & 4 & 2.6 & \\
\hline & Total & 74 & 100 & 78 & 100 & 152 & 100 & \\
\hline
\end{tabular}

Table 4:- Online Shopping Behaviour by Occupation

\begin{tabular}{|c|c|c|c|c|c|c|c|c|}
\hline \multirow{3}{*}{ Shopping Behaviour } & \multirow[b]{3}{*}{ Response } & \multicolumn{6}{|c|}{ Occupation } & \multirow{3}{*}{$\chi 2, \mathbf{p}$} \\
\hline & & \multicolumn{2}{|c|}{ Student } & \multicolumn{2}{|c|}{$\begin{array}{c}\text { Working } \\
\text { Professional }\end{array}$} & \multicolumn{2}{|c|}{ Total } & \\
\hline & & No. & $\%$ & No. & $\%$ & No. & $\%$ & \\
\hline \multirow{3}{*}{$\begin{array}{l}\text { Have you ever visited an online } \\
\text { shopping website? }\end{array}$} & Yes & 60 & 100 & 91 & 97.8 & 151 & 98.7 & \multirow{3}{*}{$\begin{array}{c}\chi 2=1.307 \\
p=0.253\end{array}$} \\
\hline & No & 0 & 0 & 2 & 2.2 & 2 & 1.3 & \\
\hline & Total & 60 & 100 & 93 & 100 & 153 & 100 & \\
\hline \multirow{6}{*}{$\begin{array}{l}\text { Frequency of your online } \\
\text { shopping }\end{array}$} & Very Low & 4 & 6.7 & 6 & 6.5 & 10 & 6.5 & \multirow{6}{*}{$\begin{array}{l}\chi^{2}=7.32 \\
p=0.198\end{array}$} \\
\hline & Low & 14 & 23.3 & 13 & 14 & 27 & 17.6 & \\
\hline & Medium & 25 & 41.7 & 30 & 32.3 & 55 & 35.9 & \\
\hline & High & 11 & 18.3 & 22 & 23.7 & 33 & 21.6 & \\
\hline & Very High & 6 & 10 & 20 & 21.5 & 26 & 17 & \\
\hline & Total & 60 & 100 & 93 & 100 & 153 & 100 & \\
\hline \multirow{3}{*}{$\begin{array}{l}\text { Do you consider returning the } \\
\text { product if there is a delay in the } \\
\text { expected delivery time? }\end{array}$} & Yes & 26 & 44.1 & 42 & 45.7 & 68 & 45 & \multirow{3}{*}{$\begin{array}{c}\chi 2=0.036 \\
p=0.849\end{array}$} \\
\hline & No & 33 & 55.9 & 50 & 54.3 & 83 & 55 & \\
\hline & Total & 59 & 100 & 92 & 100 & 151 & 100 & \\
\hline \multirow{3}{*}{ Have you bought anything online? } & Yes & 58 & 98.3 & 90 & 96.8 & 148 & 97.4 & \multirow{3}{*}{$\begin{array}{c}\chi 2=0.033 \\
\mathrm{p}=0.566\end{array}$} \\
\hline & No & 1 & 1.7 & 3 & 3.2 & 4 & 2.6 & \\
\hline & Total & 59 & 100 & 93 & 100 & 152 & 100 & \\
\hline
\end{tabular}

Factors determining online purchase:-

Four factors namely return policy, influence of social media on online shopping, special discount offer, heavy discount were analyzed to determine the factors influencing online purchase. About $4 / 5^{\text {th }}$ of respondents agreed and strongly agreed that they should know about the return policy before making any purchase. However, 'return policy' as a factor of online purchase did not have any association with the age $(\mathrm{p}=0.570)$. 
Half of the respondents agreed or strongly agreed that that social media advertisement had an influence on the online purchase. Nearly $30 \%$ disagreed or strongly disagreed with the influence of social media and about $20 \%$ were neutral about the proposition. Thus social media advertisement plays an important role in influencing online shopping behavior. However, it does not have any association with the age, gender and occupation.

Table 5:- Factors determining online purchase by Age group

\begin{tabular}{|c|c|c|c|c|c|c|c|c|c|c|}
\hline \multirow[t]{3}{*}{ Online Purchase } & \multirow[t]{3}{*}{ Response } & \multicolumn{8}{|c|}{ Age Group } & \multirow{3}{*}{$\chi 2, p$} \\
\hline & & \multicolumn{2}{|c|}{$18-25$} & \multicolumn{2}{|c|}{$26-30$} & \multicolumn{2}{|c|}{$>30$} & \multicolumn{2}{|c|}{ Total } & \\
\hline & & No. & $\%$ & No. & $\%$ & No. & $\%$ & No. & $\%$ & \\
\hline \multirow{6}{*}{$\begin{array}{l}\text { Is it important to know } \\
\text { the return policy before } \\
\text { making the purchase }\end{array}$} & Strongly Disagree & 0 & 0 & 3 & 5.6 & 2 & 5.9 & 5 & 3.5 & \multirow{6}{*}{$\begin{array}{c}\chi 2=6.697 \\
p=0.570\end{array}$} \\
\hline & Disagree & 3 & 5.7 & 4 & 7.4 & 0 & 0 & 7 & 5 & \\
\hline & Neutral & 5 & 9.4 & 6 & 11.1 & 5 & 14.7 & 16 & 11.3 & \\
\hline & Agree & 11 & 20.8 & 9 & 16.7 & 8 & 23.5 & 28 & 19.9 & \\
\hline & Strongly Agree & 34 & 64.2 & 32 & 59.3 & 19 & 55.9 & 85 & 60.3 & \\
\hline & Total & 53 & 100 & 54 & 100 & 34 & 100 & 141 & 100 & \\
\hline \multirow{6}{*}{$\begin{array}{l}\text { The social media ads } \\
\text { influence you to make } \\
\text { online purchase. }\end{array}$} & Strongly Disagree & 2 & 3.8 & 1 & 1.9 & 7 & 20.6 & 10 & 7.1 & \multirow{6}{*}{$\begin{aligned} \chi 2 & =14.301 \\
p & =0.074\end{aligned}$} \\
\hline & Disagree & 7 & 13.2 & 7 & 13.2 & 4 & 11.8 & 18 & 12.9 & \\
\hline & Neutral & 14 & 26.4 & 19 & 35.8 & 9 & 26.5 & 42 & 30 & \\
\hline & Agree & 18 & 34 & 17 & 32.1 & 7 & 20.6 & 42 & 30 & \\
\hline & Strongly Agree & 12 & 22.6 & 9 & 17 & 7 & 20.6 & 28 & 20 & \\
\hline & Total & 53 & 100 & 53 & 100 & 34 & 100 & 140 & 100 & \\
\hline \multirow{6}{*}{$\begin{array}{l}\text { Special discount offers } \\
\text { motivate you to buy } \\
\text { something or the other }\end{array}$} & Strongly Disagree & 0 & 0 & 3 & 5.6 & 4 & 11.8 & 7 & 5 & \multirow{6}{*}{$\begin{aligned} \chi 2 & =13.422 \\
p & =0.098\end{aligned}$} \\
\hline & Disagree & 5 & 9.4 & 4 & 7.4 & 5 & 14.7 & 14 & 9.9 & \\
\hline & Neutral & 12 & 22.6 & 17 & 31.5 & 10 & 29.4 & 39 & 27.7 & \\
\hline & Agree & 25 & 47.2 & 20 & 37 & 6 & 17.6 & 51 & 36.2 & \\
\hline & Strongly Agree & 11 & 20.8 & 10 & 18.5 & 9 & 26.5 & 30 & 21.3 & \\
\hline & Total & 53 & 100 & 54 & 100 & 34 & 100 & 141 & 100 & \\
\hline \multirow{3}{*}{$\begin{array}{l}\text { Did it ever happen that } \\
\text { you bought a product } \\
\text { only because of a heavy } \\
\text { discount? }\end{array}$} & Yes & 36 & 67.9 & 38 & 71.7 & 14 & 41.2 & 88 & 62.9 & \multirow{3}{*}{$\begin{array}{c}\chi^{2}=9.203 \\
p=0.010\end{array}$} \\
\hline & No & 17 & 32.1 & 15 & 28.3 & 20 & 58.8 & 52 & 37.1 & \\
\hline & Total & 53 & 100 & 53 & 100 & 34 & 100 & 140 & 100 & \\
\hline
\end{tabular}

About $56.5 \%$ opined that 'special discount offer' motivate them to (agree or strongly agree) buy something or the other while the remaining $44 \%$ did not subscribe to this factor. Age did not show any significant association with this factor $(\mathrm{p}=0.098)$. About $63 \%$ affirmed that they bought the product only because of a heavy discount. This syndrome has a significantly higher manifestation in the younger age group with $\mathrm{p}=0.010$. In the younger age group of 18-25 and 26-30 years the proportion of people who agreed or strongly agreed was around $70 \%$ while that in the age group above 30 years was about $40 \%$.

Analysis of factors determining online purchase by gender and occupation is given in tables 6 and 7. Gender and occupation do not show any association with the different factors affecting the online purchase. 
Table 6:- Factors determining online purchase by Gender.

\begin{tabular}{|c|c|c|c|c|c|c|c|c|}
\hline \multirow[t]{3}{*}{ Online Purchase } & \multirow{3}{*}{ Response } & \multicolumn{6}{|c|}{ Gender } & \multirow{3}{*}{$\chi 2, \mathbf{p}$} \\
\hline & & \multicolumn{2}{|c|}{ Male } & \multicolumn{2}{|c|}{ Female } & \multicolumn{2}{|c|}{ Total } & \\
\hline & & No. & $\%$ & No. & $\%$ & No. & $\%$ & \\
\hline \multirow{6}{*}{$\begin{array}{l}\text { Is it important to know the } \\
\text { return policy before making the } \\
\text { purchase (1. Strongly disagree; } \\
\text { 5. Strongly Agree) }\end{array}$} & Strongly Disagree & 4 & 5.4 & 4 & 5.1 & 8 & 5.2 & \multirow{6}{*}{$\begin{array}{c}\chi 2=4.404 \\
p=0.354\end{array}$} \\
\hline & Disagree & 5 & 6.8 & 3 & 3.8 & 8 & 5.2 & \\
\hline & Neutralk & 11 & 14.9 & 5 & 6.3 & 16 & 10.5 & \\
\hline & Agree & 11 & 14.9 & 17 & 21.5 & 28 & 18.3 & \\
\hline & Strongly Agree & 43 & 58.1 & 50 & 63.3 & 93 & 60.8 & \\
\hline & Total & 74 & 100 & 79 & 100 & 153 & 100 & \\
\hline \multirow{6}{*}{$\begin{array}{l}\text { The social media ads influence } \\
\text { you to make online purchase. }\end{array}$} & Strongly Disagree & 10 & 13.5 & 3 & 3.8 & 13 & 8.6 & \multirow{6}{*}{$\begin{array}{c}\chi 2=4.824 \\
\mathrm{p}=0.306\end{array}$} \\
\hline & Disagree & 9 & 12.2 & 11 & 14.1 & 20 & 13.2 & \\
\hline & Neutral & 20 & 27 & 24 & 30.8 & 44 & 28.9 & \\
\hline & Agree & 23 & 31.1 & 24 & 30.8 & 47 & 30.9 & \\
\hline & Strongly Agree & 12 & 16.2 & 16 & 20.5 & 28 & 18.4 & \\
\hline & Total & 74 & 100 & 78 & 100 & 152 & 100 & \\
\hline \multirow{6}{*}{$\begin{array}{c}\text { special discount offers } \\
\text { motivate you to buy something } \\
\text { or the other }\end{array}$} & Strongly Disagree & 5 & 6.8 & 3 & 3.8 & 8 & 5.2 & \multirow{6}{*}{$\begin{array}{l}\chi 2=0.99 \\
\mathrm{p}=0.911\end{array}$} \\
\hline & Disagree & 8 & 10.8 & 9 & 11.4 & 17 & 11.1 & \\
\hline & Neutral & 18 & 24.3 & 22 & 27.8 & 40 & 26.1 & \\
\hline & Agree & 26 & 35.1 & 29 & 36.7 & 55 & 35.9 & \\
\hline & Strongly Agree & 17 & 23 & 16 & 20.3 & 33 & 21.6 & \\
\hline & Total & 74 & 100 & 79 & 100 & 153 & 100 & \\
\hline \multirow{3}{*}{$\begin{array}{c}\text { Did it ever happen that you } \\
\text { bought a product only because } \\
\text { of a heavy discount? }\end{array}$} & Yes & 48 & 65.8 & 48 & 60.8 & 96 & 63.2 & \multirow{3}{*}{$\begin{array}{c}\chi 2=0.407 \\
p=0.524\end{array}$} \\
\hline & No & 25 & 34.2 & 31 & 39.2 & 56 & 36.8 & \\
\hline & Total & 73 & 100 & 79 & 100 & 152 & 100 & \\
\hline
\end{tabular}

Table 7:- Factors determining online purchase by Occupation

\begin{tabular}{|c|c|c|c|c|c|c|c|c|}
\hline \multirow[t]{3}{*}{ Online Purchase } & \multirow[t]{3}{*}{ Response } & \multicolumn{6}{|c|}{ Occupation } & \multirow{3}{*}{$\chi 2, \mathbf{p}$} \\
\hline & & \multicolumn{2}{|c|}{ Student } & \multicolumn{2}{|c|}{$\begin{array}{c}\text { Working } \\
\text { Professional }\end{array}$} & \multicolumn{2}{|c|}{ Total } & \\
\hline & & No. & $\%$ & No. & $\%$ & No. & $\%$ & \\
\hline \multirow{6}{*}{$\begin{array}{l}\text { Is it important to know the } \\
\text { return policy before making the } \\
\text { purchase }\end{array}$} & Strongly Disagree & 2 & 3.3 & 6 & 6.5 & 8 & 5.2 & \multirow{6}{*}{$\begin{array}{c}\chi^{2}=1.3 \\
29 \\
\mathrm{p}=0.85 \\
6\end{array}$} \\
\hline & Disagree & 3 & 5 & 5 & 5.4 & 8 & 5.2 & \\
\hline & Neutral & 6 & 10 & 10 & 10.8 & 16 & 10.5 & \\
\hline & Agree & 13 & 21.7 & 15 & 16.1 & 28 & 18.3 & \\
\hline & Strongly Agree & 36 & 60 & 57 & 61.3 & 93 & 60.8 & \\
\hline & Total & 60 & 100 & 93 & 100 & 153 & 100 & \\
\hline \multirow{6}{*}{$\begin{array}{l}\text { The social media ads influence } \\
\text { you to make online purchase. }\end{array}$} & Strongly Disagree & 3 & 5 & 10 & 10.9 & 13 & 8.6 & \multirow{6}{*}{$\begin{array}{c}\chi^{2}=3.3 \\
11 \\
\mathrm{p}=0.50 \\
7\end{array}$} \\
\hline & Disagree & 6 & 10 & 14 & 15.2 & 20 & 13.2 & \\
\hline & Neutral & 18 & 30 & 26 & 28.3 & 44 & 28.9 & \\
\hline & Agree & 22 & 36.7 & 25 & 27.2 & 47 & 30.9 & \\
\hline & Strongly Agree & 11 & 18.3 & 17 & 18.5 & 28 & 18.4 & \\
\hline & Total & 60 & 100 & 92 & 100 & 152 & 100 & \\
\hline \multirow{6}{*}{$\begin{array}{l}\text { special discount offers } \\
\text { motivate you to buy something } \\
\text { or the other }\end{array}$} & Strongly Disagree & 0 & 0 & 8 & 8.6 & 8 & 5.2 & \multirow{6}{*}{$\begin{array}{c}\chi^{2}=6.2 \\
1 \\
p=0.18 \\
4\end{array}$} \\
\hline & Disagree & 7 & 11.7 & 10 & 10.8 & 17 & 11.1 & \\
\hline & Neutral & 16 & 26.7 & 24 & 25.8 & 40 & 26.1 & \\
\hline & Agree & 25 & 41.7 & 30 & 32.3 & 55 & 35.9 & \\
\hline & Strongly Agree & 12 & 20 & 21 & 22.6 & 33 & 21.6 & \\
\hline & Total & 60 & 100 & 93 & 100 & 153 & 100 & \\
\hline \multirow{3}{*}{$\begin{array}{l}\text { Did it ever happen that you } \\
\text { bought a product only because } \\
\text { of a heavy discount? }\end{array}$} & Yes & 41 & 68.3 & 55 & 59.8 & 96 & 63.2 & \multirow{3}{*}{$\begin{array}{c}\chi 2=1.1 \\
41 \\
p=0.28 \\
5\end{array}$} \\
\hline & No & 19 & 31.7 & 37 & 40.2 & 56 & 36.8 & \\
\hline & Total & 60 & 100 & 92 & 100 & 152 & 100 & \\
\hline
\end{tabular}




\section{Perception about online shopping:-}

In the study the respondents were asked to furnish their perception on different aspects of online shopping. These aspects were 'effect of online shopping on retail shops', 'pricing of the product, 'quality of product' 'delivery of product', and 'payment method'. The analysis is showcased in tables 8, 9 and 10.

Table 8:- Perception about online shopping by Age group.

\begin{tabular}{|c|c|c|c|c|c|c|c|c|c|c|}
\hline \multirow[t]{3}{*}{ Online Purchase } & \multirow[t]{3}{*}{ Response } & \multicolumn{8}{|c|}{ Age Group } & \multirow{3}{*}{$\chi 2, p$} \\
\hline & & \multicolumn{2}{|c|}{$18-25$} & \multicolumn{2}{|c|}{$26-30$} & \multicolumn{2}{|c|}{$>30$} & \multicolumn{2}{|c|}{ Total } & \\
\hline & & No. & $\%$ & No. & $\%$ & No. & $\%$ & No. & $\%$ & \\
\hline \multirow{6}{*}{$\begin{array}{l}\text { Offline retail shops are } \\
\text { getting affected by the } \\
\text { increasing popularity of } \\
\text { online shopping? }\end{array}$} & Strongly Disagree & 1 & 1.9 & 2 & 3.8 & 2 & 5.9 & 5 & 3.6 & \multirow{6}{*}{$\begin{array}{c}\chi^{2}=5.283 \\
p=0.727\end{array}$} \\
\hline & Disagree & 6 & 11.5 & 6 & 11.3 & 6 & 17.6 & 18 & 12.9 & \\
\hline & Neutral & 14 & 26.9 & 20 & 37.7 & 10 & 29.4 & 44 & 31.7 & \\
\hline & Agreek & 21 & 40.4 & 13 & 24.5 & 9 & 26.5 & 43 & 30.9 & \\
\hline & Strongly Agree & 10 & 19.2 & 12 & 22.6 & 7 & 20.6 & 29 & 20.9 & \\
\hline & Total & 52 & 100 & 53 & 100 & 34 & 100 & 139 & 100 & \\
\hline \multirow{5}{*}{$\begin{array}{l}\text { Rating of Pricing of the } \\
\text { products }\end{array}$} & Very Dissatisfied & 2 & 3.9 & 2 & 3.8 & 2 & 5.9 & 6 & 4.4 & \multirow{5}{*}{$\begin{array}{c}\chi^{2}=3.781 \\
p=0.706\end{array}$} \\
\hline & Dissatisfied & 1 & 2 & 4 & 7.7 & 1 & 2.9 & 6 & 4.4 & \\
\hline & Satisfied & 38 & 74.5 & 39 & 75 & 23 & 67.6 & 100 & 73 & \\
\hline & Very Satisfied & 10 & 19.6 & 7 & 13.5 & 8 & 23.5 & 25 & 18.2 & \\
\hline & Total & 51 & 100 & 52 & 100 & 34 & 100 & 137 & 100 & \\
\hline \multirow{5}{*}{$\begin{array}{l}\text { Rating of Quality of } \\
\text { products }\end{array}$} & Very Dissatisfied & 2 & 3.9 & 2 & 3.9 & 2 & 5.9 & 6 & 4.4 & \multirow{5}{*}{$\begin{array}{c}\chi 2=5.197 \\
p=0.519\end{array}$} \\
\hline & Dissatisfied & 8 & 15.7 & 3 & 5.9 & 5 & 14.7 & 16 & 11.8 & \\
\hline & Satisfied & 33 & 64.7 & 42 & 82.4 & 23 & 67.6 & 98 & 72.1 & \\
\hline & Very Dissatisfied & 8 & 15.7 & 4 & 7.8 & 4 & 11.8 & 16 & 11.8 & \\
\hline & Total & 51 & 100 & 51 & 100 & 34 & 100 & 136 & 100 & \\
\hline \multirow{5}{*}{$\begin{array}{l}\text { Rating of Payment } \\
\text { method }\end{array}$} & Very Dissatisfied & 3 & 5.9 & 3 & 5.9 & 2 & 5.9 & 8 & 5.9 & \multirow{5}{*}{$\begin{array}{c}\chi 2=3.024 \\
p=0.806\end{array}$} \\
\hline & Dissatisfied & 2 & 3.9 & 1 & 2 & 0 & 0 & 3 & 2.2 & \\
\hline & Satisfied & 26 & 51 & 30 & 58.8 & 16 & 47.1 & 72 & 52.9 & \\
\hline & Very Satisfied & 20 & 39.2 & 17 & 33.3 & 16 & 47.1 & 53 & 39 & \\
\hline & Total & 51 & 100 & 51 & 100 & 34 & 100 & 136 & 100 & \\
\hline \multirow[t]{5}{*}{ Rating of Delivery } & Very Dissatisfied & 2 & 3.9 & 2 & 3.9 & 2 & 5.9 & 6 & 4.4 & \multirow{5}{*}{$\begin{array}{r}\chi^{2}=5.547 \\
p=0.476\end{array}$} \\
\hline & Dissatisfied & 8 & 15.7 & 2 & 3.9 & 2 & 5.9 & 12 & 8.8 & \\
\hline & Satisfied & 27 & 52.9 & 33 & 64.7 & 22 & 64.7 & 82 & 60.3 & \\
\hline & Very Satisfied & 14 & 27.5 & 14 & 27.5 & 8 & 23.5 & 36 & 26.5 & \\
\hline & Total & 51 & 100 & 51 & 100 & 34 & 100 & 136 & 100 & \\
\hline
\end{tabular}

Table 9:- Perception about online shopping by Gender.

\begin{tabular}{|c|c|c|c|c|c|c|c|c|}
\hline \multirow{3}{*}{ Online Purchase } & \multirow{3}{*}{ Response } & \multicolumn{6}{|c|}{ Gender } & \multirow{3}{*}{$\chi 2, \mathbf{p}$} \\
\hline & & \multicolumn{2}{|c|}{ Male } & \multicolumn{2}{|c|}{ Female } & \multicolumn{2}{|c|}{ Total } & \\
\hline & & No. & $\%$ & No. & $\%$ & No. & $\%$ & \\
\hline \multirow{6}{*}{$\begin{array}{l}\text { Offline retail shops are } \\
\text { getting affected by the } \\
\text { increasing popularity of } \\
\text { online shopping? }\end{array}$} & Strongly Dissatisfied & 3 & 4.1 & 2 & 2.6 & 5 & 3.3 & \multirow{6}{*}{$\begin{aligned} \chi 2 & =3.551 \\
p & =0.470\end{aligned}$} \\
\hline & Disagree & 6 & 8.2 & 14 & 17.9 & 20 & 13.2 & \\
\hline & Neutral & 24 & 32.9 & 26 & 33.3 & 50 & 33.1 & \\
\hline & Agree & 24 & 32.9 & 21 & 26.9 & 45 & 29.8 & \\
\hline & Strongly Agree & 16 & 21.9 & 15 & 19.2 & 31 & 20.5 & \\
\hline & Total & 73 & 100 & 78 & 100 & 151 & 100 & \\
\hline \multirow{3}{*}{$\begin{array}{l}\text { Please rate the following } \\
\text { aspects about online } \\
\text { shopping. [Pricing of the } \\
\text { products] }\end{array}$} & Very Dissatisfied & 3 & 4.2 & 3 & 3.9 & 6 & 4 & \multirow{3}{*}{$\begin{array}{c}\chi^{2}=6.639 \\
p=0.084\end{array}$} \\
\hline & Dissatisfied & 1 & 1.4 & 5 & 6.5 & 6 & 4 & \\
\hline & Satisfied & 51 & 70.8 & 61 & 79.2 & 112 & 75.2 & \\
\hline
\end{tabular}




\begin{tabular}{|c|c|c|c|c|c|c|c|c|}
\hline & Very Satisfied & 17 & 23.6 & 8 & 10.4 & 25 & 16.8 & \\
\hline & Total & 72 & 100 & 77 & 100 & 149 & 100 & \\
\hline & Very Dissatisfied & 3 & 4.2 & 3 & 3.9 & 6 & 4.1 & \\
\hline & Dissatisfied & 5 & 6.9 & 11 & 14.5 & 16 & 10.8 & \\
\hline Rating of Quality of products & Satisfied & 53 & 73.6 & 57 & 75 & 110 & 74.3 & $\begin{array}{c}\chi 2=4.541 \\
p=0.209\end{array}$ \\
\hline & Very Satisfied & 11 & 15.3 & 5 & 6.6 & 16 & 10.8 & \\
\hline & Total & 72 & 100 & 76 & 100 & 148 & 100 & \\
\hline & Very Dissatisfied & 4 & 5.6 & 4 & 5.3 & 8 & 5.4 & \\
\hline & Dissatisfied & 0 & 0 & 3 & 3.9 & 3 & 2 & \\
\hline Rating of Payment method & Satisfied & 35 & 48.6 & 46 & 60.5 & 81 & 54.7 & $\chi^{2}=6.176$ \\
\hline & Very Satisfied & 33 & 45.8 & 23 & 30.3 & 56 & 37.8 & \\
\hline & Total & 72 & 100 & 76 & 100 & 148 & 100 & \\
\hline & Very Dissatisfied & 3 & 4.2 & 3 & 3.9 & 6 & 4.1 & \\
\hline & Dissatisfied & 4 & 5.6 & 8 & 10.5 & 12 & 8.1 & \\
\hline Rating of Delivery & Satisfied & 45 & 62.5 & 47 & 61.8 & 92 & 62.2 & $\begin{array}{c}\chi 2=1.375 \\
p=0.711\end{array}$ \\
\hline & Very Satisfied & 20 & 27.8 & 18 & 23.7 & 38 & 25.7 & \\
\hline & Total & 72 & 100 & 76 & 100 & 148 & 100 & \\
\hline
\end{tabular}

Table 10:- Perception about online shopping by Occupation.

\begin{tabular}{|c|c|c|c|c|c|c|c|c|}
\hline \multirow{3}{*}{ Online Purchase } & & \multicolumn{6}{|c|}{ Occupation } & \multirow{3}{*}{$\chi 2, \mathbf{p}$} \\
\hline & & \multicolumn{2}{|c|}{ Student } & \multicolumn{2}{|c|}{$\begin{array}{c}\text { Working } \\
\text { Professional }\end{array}$} & \multicolumn{2}{|c|}{ Total } & \\
\hline & & No. & $\%$ & No. & $\%$ & No. & $\%$ & \\
\hline \multirow{6}{*}{$\begin{array}{l}\text { Offline retail shops are } \\
\text { getting affected by the } \\
\text { increasing popularity of } \\
\text { online shopping? }\end{array}$} & Strongly Disagree & 1 & 1.7 & 4 & 4.3 & 5 & 3.3 & \multirow{6}{*}{$\begin{array}{c}\chi^{2}=2.23 \\
4 \\
p=0.693\end{array}$} \\
\hline & Disagree & 9 & 15.3 & 11 & 12 & 20 & 13.2 & \\
\hline & Neutral & 19 & 32.2 & 31 & 33.7 & 50 & 33.1 & \\
\hline & Agree & 20 & 33.9 & 25 & 27.2 & 45 & 29.8 & \\
\hline & Strongly Agree & 10 & 16.9 & 21 & 22.8 & 31 & 20.5 & \\
\hline & Total & 59 & 100 & 92 & 100 & 151 & 100 & \\
\hline \multirow{5}{*}{$\begin{array}{l}\text { Please rate the following } \\
\text { aspects about online } \\
\text { shopping. [Pricing of the } \\
\text { products] }\end{array}$} & Very Dissatisfied & 2 & 3.4 & 4 & 4.4 & 6 & 4 & \multirow{5}{*}{$\begin{array}{c}\chi^{2}=0.43 \\
4 \\
p=0.933\end{array}$} \\
\hline & Dissatisfied & 2 & 3.4 & 4 & 4.4 & 6 & 4 & \\
\hline & Satisfied & 46 & 78 & 66 & 73.3 & 112 & 75.2 & \\
\hline & Very Satisfied & 9 & 15.3 & 16 & 17.8 & 25 & 16.8 & \\
\hline & Total & 59 & 100 & 90 & 100 & 149 & 100 & \\
\hline \multirow{5}{*}{$\begin{array}{l}\text { Rating of Quality of } \\
\text { products }\end{array}$} & Very Dissatisfied & 2 & 3.4 & 4 & 4.4 & 6 & 4.1 & \multirow{5}{*}{$\begin{array}{l}\chi 2=1.18 \\
p=0.758\end{array}$} \\
\hline & Dissatisfied & 7 & 12.1 & 9 & 10 & 16 & 10.8 & \\
\hline & Satisfied & 41 & 70.7 & 69 & 76.7 & 110 & 74.3 & \\
\hline & Very Satisfied & 8 & 13.8 & 8 & 8.9 & 16 & 10.8 & \\
\hline & Total & 58 & 100 & 90 & 100 & 148 & 100 & \\
\hline \multirow[t]{5}{*}{ Rating of Payment method } & Very Dissatisfied & 3 & 5.2 & 5 & 5.6 & 8 & 5.4 & \multirow{5}{*}{$\begin{array}{c}\chi^{2}=0.05 \\
6 \\
p=0.996\end{array}$} \\
\hline & Dissatisfied & 1 & 1.7 & 2 & 2.2 & 3 & 2 & \\
\hline & Satisfied & 32 & 55.2 & 49 & 54.4 & 81 & 54.7 & \\
\hline & Very Satisfied & 22 & 37.9 & 34 & 37.8 & 56 & 37.8 & \\
\hline & Total & 58 & 100 & 90 & 100 & 148 & 100 & \\
\hline \multirow[t]{5}{*}{ Rating of Delivery } & Very Dissatisfied & 2 & 3.4 & 4 & 4.4 & 6 & 4.1 & \multirow{5}{*}{$\begin{array}{c}\chi^{2}=3.17 \\
2 \\
p=0.366\end{array}$} \\
\hline & Dissatisfied & 7 & 12.1 & 5 & 5.6 & 12 & 8.1 & \\
\hline & Satisfied & 32 & 55.2 & 60 & 66.7 & 92 & 62.2 & \\
\hline & Very Satisfied & 17 & 29.3 & 21 & 23.3 & 38 & 25.7 & \\
\hline & Total & 58 & 100 & 90 & 100 & 148 & 100 & \\
\hline
\end{tabular}


About 52\% respondents agreed or strongly agreed that offline retail shops are getting affected by the increasing popularity of online shopping, 32\% neither agreed nor disagreed and about 16\% disagreed with the above. Views of the respondents did not show any significant association with the age groups that is, the opinion is independent of age group. About $91 \%$ expressed satisfaction with the pricing of the products. This was more or less uniform across the age groups $(\mathrm{p}=0.706)$. As regards quality of the product, about $84 \%$ respondents expressed their satisfaction and the opinion is independent of the age group $(\mathrm{p}=0.519)$. About $92 \%$ respondents rated the payment method as satisfactory (satisfied or very satisfied). This view is more or less homogeneous across the age groups ( $\mathrm{p}=0.806$ ). About $87 \%$ of the respondents were satisfied or very satisfied regarding the delivery of the products through online shopping. This view is more or less same across the age groups. Table 9 and 10 show the perception of the respondents on the four aspects discussed above with respect to their association with gender and occupation revealing that the perception did not vary significantly across gender or occupation groups.

The open ended question on whether they faced any difficulty while shopping online fetched 120 responses. Out of that 71 reacted with a clear "no" and 8 with a "yes". The remaining 41 specified the difficulty faced which were as follows: 10 out of the 41 complained of delayed delivery or goods not delivered to certain areas; 7 out of 41 complained of unfriendly return policy or delay in the process; 4 reported inferior quality of product as compared to the image and description displayed on the screen; Size was an issue with 7 respondents as the size chart for the product was misleading and the UK to Indian size conversion was not correct; Similarly 4 complained about variance in colour as compared to what was exhibited and 4 about the product not matching the description mentioned on the website; Anxiety about the genuineness of the product and doubt about the authenticity of the same fetched only 3 responses; Again only 2 objected to many choices/options causing confusion and making the website appear too cluttered.

The other open ended question requesting suggestions for a better online shopping experience gathered only 104 responses where $70 \%$ thought it was good as it is and 30\% of the respondents suggested faster delivery and accessibility to more areas, more information about product, videos showing the product and user friendly websites with exact product description, easy return policy within a stipulated period of time, cash on delivery facility required for all products, accurate size charts and genuine product assurance to be given by portal. Apart from this some praise and criticism regarding various online shopping portals like Flipcart, Snapdeal, Jabong and Amazon was voiced, for instance, "Amazon is simply awesome in the 360 degree mode" which could open many avenues for future research. Similarly the peeves and recommendations regarding online shopping could be indicators for improvement in the future. Moreover, these two questions inviting the respondent's comments on problems and suggestions did not include a single mention of either pricing or discounts indicating satisfaction with both.

Besides this , frequency of items purchased by respondents depicted in Fig. 1 in the following section revealed that, apparel is the most common shopping item followed by electronic items and accessories.

\section{Discussion:-}

The fact that discounts available online have fared marginally better with $70.6 \%$ votes than convenience (which has $66.3 \%$ votes and has always been considered as the supreme reason for choosing online over offline shopping), it does indicate that discount is the prime motivating factor. Almost $60 \%$ agree that special discounts motivate to buy something or the other thereby revealing the underlying clause (even if you don't need it). Further $62.3 \%$ have bought a product only because of a heavy discount again pointing to the possibility of compulsive buying whether or not the product was really required. Moreover, pricing comes across as a winner as $92.2 \%$ express satisfaction with the pricing of products. $93.5 \%$ are satisfied with the payment method.

Billy Hulkower, Senior Technology Analyst at Mintel opines that apparel, accessories and electronics are "among the products most commonly purchased online" and are also the "prime candidates for impulse buying, but at the same time, do not generally fulfill immediate needs". In the present study too we have discovered that these three categories are the most preferred for online shopping corroborating the above statement. If these are the chief culprits of "impulsive buying" and they are being purchased the most, then online shopping is probably a good breeding ground for compulsive buying. Frequency of items purchased by respondents in Fig. 1 revealed that, apparel is the most common shopping item followed by electronic items and accessories. 


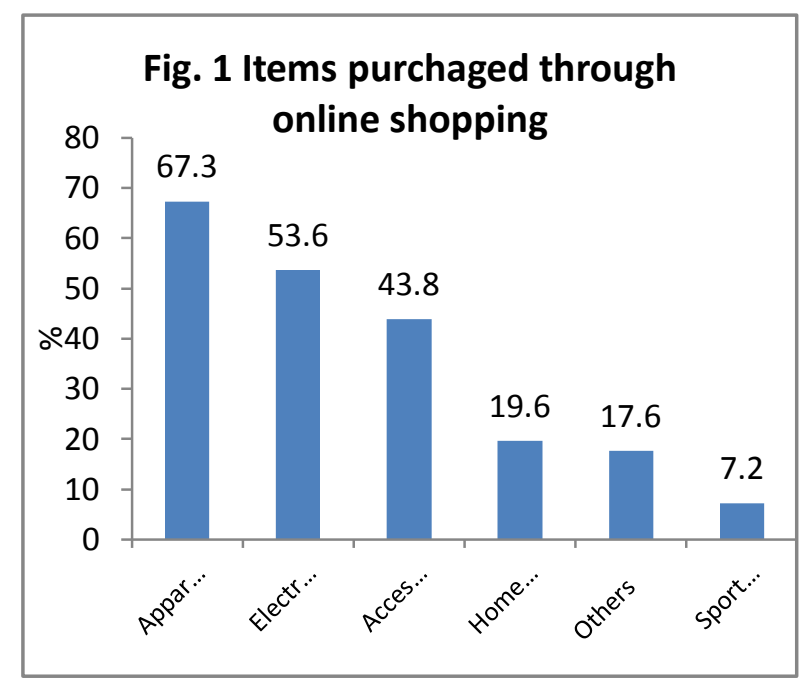

The senior analyst further adds that "online women's apparel and footwear purchases increased in 2015, suggesting that shoppers, particularly women, are warming to the idea that they can try clothes on at home and simply return products they don't want" which actually is a shift from the earlier research which says that men have a more "positive attitude" towards online shopping than women.

\section{Conclusion:-}

The soaring frequency of online shopping and a whopping $97.1 \%$ of the respondents purchasing online herald a new era of buying which is here to stay. Convenience, which is definitely a major factor in prompting online shopping has a close contender racing against it called special discounts and reduced cost as compared to the in-store price which includes the additional operational frills. Similarly with apparel emerging as the leading category of products bought online followed by electronics and accessories, online shopping becomes a potent platform for compulsive buying.

\section{References:-}

1. Abraham, Magid, 2008, The Off-Line Impact of Online Ads, Harvard Business Review https://hbr.org/2008/04/the-off-line-impact-of-online-ads, Accessed on 13 June 2016

2. Cho, H., and S. K. Jialin, 2008, Influence of gender on Internet commerce: An explorative study in Singapore, Journal of Internet Commerce 7 (1): 95-119.

3. Chou, C., H.-C. Wu, and C.-H. Chen, 2010, Re-visiting college students' attitudes toward the Internet-based on a 6-T model: Gender and grade level difference, Computers and Education 56:939-947

4. Dittmar H., Long K., Meek R., 2004, Buying on the Internet: Gender differences in online and conventional buying motivations. Sex Roles, 50(5-6):423-444.

5. Dittmar H., 2005, Compulsive buying - a growing concern? An examination of gender, age, and endorsement of materialistic values as predictors. British Journal of Psychology, 96(4):467-491.

6. Dittmar H., Long K., Bond R., 2007, When a better self is only a button click away: Associations between materialistic values, emotional and identity-related buying motives, and compulsive buying tendency online. Journal of Social and Clinical Psychology, 26(3):334-361.

7. Eastin, Matthew S., 2002, Diffusion of E-Commerce: An Analysis of the Adoption of Four E-Commerce Activities, Telematics and Infomatics, 19 (3), 251-67.

8. Faber, Ronald J., Thomas C. O'Guinn, and Raymond Krych, 1987, Compulsive Consumption, Advances in Consumer Research, Vol. 14, ed. Melanie Wallendorf and

9. Grant Thronton, 2015, Law and technology: Evolving challenges as a result of fraud in E-commerce sector, http://www.grantthornton.in/insights/articles/law--technology-evolving-challenges-as-a-result-of-fraud-in-ecommerce-sector, Accessed on 15 May 2016

10. Haque, H., A. K. Tarofder, S. A. Mahmud, and A. Z. Ismail, 2007, Internet advertisement in Malaysia: A study on attitudinal differences, The Electronic Journal on Information Systems in Developing Countries 31 (9): 1-15. 
11. Han Y. J., Nunes J. C., Drèze X., 2010, Signaling status with luxury goods: The role of brand prominence.Journal of Marketing, 74:15-30.

12. Hasan, B. 2010, Exploring gender differences in online shopping attitude, Computers in Human Behavior, 26:597-601.

13. Hashim, A., E. K. Ghani, and J. Said, 2009, Does consumers' demographic profile influence online shopping? An examination using Fishbein's Theory, Canadian Social Science 5 (6): 19-31.

14. Lunn, R. J., and M. W. Suman., 2002, Experience and trust in online shopping. In The internet in everyday life, ed. B. Wellman and C. Haythornthwaite, 549-577. Oxford: Blackwell Publishing.

15. Millan E. S., Howard E., 2007, Shopping for pleasure? Shopping experiences of Hungarian consumers. International Journal of Retail \& Distribution Management, 35(6):474-487.

16. Mintel's Online Shopping US 2015 report (http://www.mintel.com/press-centre/technology-press-centre/nearly70-of-americans-shop-online-regularly-with-close-to-50-taking-advantage-of-free-shipping)

17. Mintel's Online Shopping US 2015 report (http://www.mintel.com/press-centre/technology-press-centre/nearly70-of-americans-shop-online-regularly-with-close-to-50-taking-advantage-of-free-shipping)

18. Neuner M., Raab G., Reisch L. A., 2005, Compulsive buying in maturing consumer societies: An empirical reinquiry. Journal of Economic Psychology, 26(4):509-522.

19. Newsroom, Accenture, 2013, Accenture Study Shows U.S. Consumers Want a Seamless Shopping Experience Across Store, Online and Mobile that Many Retailers are Struggling to Deliver, https://newsroom.accenture.com/ industries/retail/accenture-study-shows-us-consumers-want-a-seamlessshopping-experience-across-store-online-and-mobile-that-many-retailers-are-struggling-to-deliver.htm, Accessed on 2 August 2016

20. O’Guinn T. C., Faber R. J., 1989, Compulsive buying: A phenomenological exploration. Journal of Consumer Research, 16(2):147-157.

21. Panda, Rajesh and Swar, Biranchi Narayan, Online Shopping: An Exploratory Study to Identify the Determinants of Shopper Buying Behaviour

22. Paul F. Anderson, Provo, UT: Association for Consumer Research, 132-35.

23. Ridgway N. M., Kukar-Kinney M., Monroe K. B., 2008, An expanded conceptualization and a new measure of compulsive buying, Journal of Consumer Research, 35(4):622-639.

24. Sim, L. L., and Koi, S. M., 2002, Singapore's Internet shoppers and their impact on traditional shopping patterns. Journal of Retailing and Consumer Services, 9:115-124.

25. Wei, Marlynn M.D., J.D. 2015 www.psychologytoday.com/blog/urban-survival/201511/10-signs-you-readdicted-online-shopping.

26. Yun Jung Lee and JungKun Park, 2008, The Mediating Role of Consumer Conformity in E-Compulsive Buying, Advances in Consumer Research Volume 35, eds. Angela Y. Lee and Dilip Soman, Duluth, MN : Association for Consumer Research, Pages: 387-392. 